\title{
A New Approach for the Preparation of Functional Poly(acrylonitrile-g-glycidyl methacrylate) Polymer Brush
}

\author{
Yating Wang, Wenxiang Wang, Hou Chen *, Liangjiu Bai * and Hui Xu \\ School of Chemistry and Materials Science; Ludong University; Yantai 264025, China \\ * Correspondence: lduchenhou@126.com (H.C.); bailiangjiu@163.com (L.B.); Tel.: +86-535-6697933 (H.C.); \\ $+86-535-6697933$ (L.B.)
}

\begin{abstract}
A new approach for the preparation of polymer brush was developed via the union of controlled/"living" radical polymerization and click chemistry. The application of the approach realized the more quickly and accurate design and synthesis of the polymer brushes. A novel functional polymer brush of poly(acrylonitrile-g-glycidyl methacrylate) with tremendous application potential in the field of biology has been successfully synthesized via the new approach. The reaction conditions of click chemistry were optimized with the reaction time and the reactant ratio and judged from UV-vis spectra. Under the optimum of click chemistry, the novel functional polymer brushes were prepared. The GPC, FTIR analyses, ${ }^{1} \mathrm{H}$ NMR spectrum and TGA were employed to ensure the successful synthesis of poly(acrylonitrile-g-glycidyl methacrylate) polymer brushes.
\end{abstract}

Keywords: controlled/"living" radical polymerization (CRP); polymer brush; poly(acrylonitrile)

\section{Introduction}

Polymer brushes,[1-3] defined as polymer chains attached one end to a surface or interface, generally were widely utilized to medical implants, drug delivery, biosensor and diagnostics. The common types of synthesis for polymer brushes include "graft through" , "graft from" and "graft onto". "Graft through" refers to the approach of polymerization of macromonomers, which ensures complete grafting, but it's weak to control the length of backbone. [4-8] The "graft from" technique, is knows as surface-initiated polymerization, uses initiation sites of polymer backbone to generate polymer of side-chain monomers.[9-11] The polymer backbone was well-controlled while it's insufficient for the controlling of the side-chain polymer. The "grafting onto" method involves generate polymer brushes via the reaction between the polymer backbone and the polymer as side chain. [12-14] However, the method is limited to the grafting density due to the low efficiency of functional group and the steric congestion of side chain.

Controlled/“living” radical polymerization (CRP)[15-16] has become the most common polymerization process instead of traditional free radical polymerization due to its facile reaction condition and robust control for the molecular weight of polymer. Among common controlled/"living" radical polymerizations, both atom transfer radical polymerization (ATRP) [17-23] and reversible addition-fragmentation chain transfer polymerization (RAFT)[24-28] are developed rapidly in recent years. ATRP is regarded as a distinctive approach in the preparation of macrocyclic precursors due to its application to modify the terminal end group of polymer. RAFT polymerization has been in the focus of the polymerization because of its facile reaction condition and robust control even almost quantitative end functionalization by the chain transfer agents. The combination of CRP and click chemistry provides a new grafting strategy for the design of polymer brushes. Matyjaszewski has successfully prepared three-arm and four-arm star polystyrene (PS) polymers by a combination of atom transfer radical polymerization (ATRP) and click chemistry[29]. Lutz has synthesized functional PEG analogues and PEGylated oligopeptides via the combination 
of ATRP and click chemistry[30]. Rajesh Ranjan and William J. Brittain have modified the surface of silica particles with polystyrene (PS) by employing RAFT polymerization and click chemistry[31].

Click chemistry has been widely used as a novel green chemistry in recent years[32-40]. click chemistry provides an extensively approach to quickly synthesized new polymer brushes in highly yields without special purification and serious reaction condition. The Huisgen 1,3-dipolar cycloaddition of azides and terminal alkynes with $\mathrm{Cu}(\mathrm{I})$ as catalyst is one of the most common Click reactions[41-43]. It is thought of the cream of the crop in click chemistry. Nevertheless, the application of click chemistry between azides and terminal alkynes in the synthesis of polymer brushes is limited because of the side reaction during the introduction of terminal alkynes. The click chemistry between nitrile group and azide group rose in response to the proper time and conditions. It can not only avoid the side reaction during the introduction of terminal alkynes but also greatly increase reaction efficiency of functional group.

The particular properties of polyacrilonitrile (PAN) [44], such as good thermal and mechanical stability, high tensile strength and high surface area, were attributed to the application of many fields such as fibers, film and membrane. Herein, we reported the preparation of a novel functional polymer brush of poly(acrylonitrile-g-glycidyl methacrylate) via the union of CRP and click chemistry (Scheme 1). In addition, a click chemistry reaction with sodium azide as the source of azide and ammonium chloride as catalyst was employed to modify PAN under the optimized reaction condition. The substitution reaction of bromide and tetrazole was carried out as the crucial and final step for the preparation of polymer brushes.

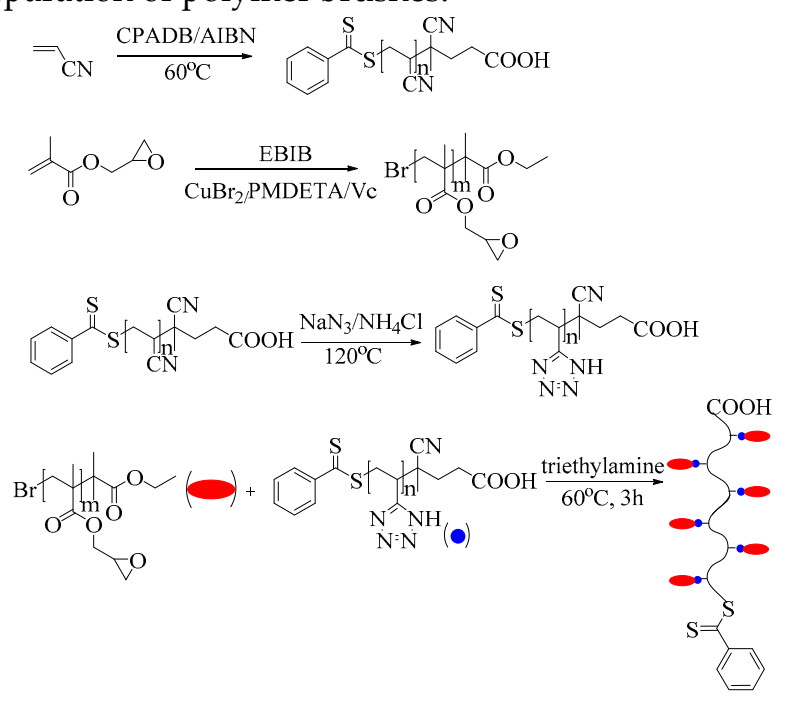

Scheme 1. Synthetic pathway for the functional polymer brushes of PAN-g-PGMA.

\section{Materials and Methods}

\section{Reagents and Materials}

Materials and Methods should be described with sufficient details to allow others to replicate and build on published results. Please note that publication of your manuscript implicates that you must make all materials, data, computer code, and protocols associated with the publication available to readers. Please disclose at the submission stage any restrictions on the availability of materials or information. New methods and protocols should be described in detail while well-established methods can be briefly described and appropriately cited.

The monomers, acrylonitrile (AN, Tianjin Fuchen Chemical Reagent Factory, China) and glycidyl methacrylate (GMA, Chengdu Aikeda Chemical Reagent Corporation, China) were all purified via simple distillation under normal pressure and stored in low temperature for use. Azobis-isobutyronitrile (AIBN) was recrystallized in ethyl alcohol for the purification. Other chemicals were used as received as well. Both $N, N$-dimethylformamide (DMF, analytical grade) and copper bromide $\left(\mathrm{CuBr}_{2}\right)$ were obtained from Tianjin Fuchen Chemical Reagent Company. 
Sodium azide $\left(\mathrm{NaN}_{3}\right)$ was purchased from Chengdu Maikaxi Chemical Reagents. Ammonium chloride $\left(\mathrm{NH}_{4} \mathrm{Cl}\right)$ and triethylamine were all obtained from Sinopharm Chemical Reagent Company. Sodium ascorbate (Vc, $\geqslant 99.7 \%$ ) was purchased from Tianjin Bodi Chemical Company. 4-Cyanopentanoic Acid Dithiobenzoate (CPADB) was prepared according to the approach reported [45]. 1,1,4,7,7-Pentamethyldiethylenetriamine (PMDETA, $\geqslant 98 \%$, Beijing HWRK Chemical Company), ethyl a-bromoisobutyrate (EBIB, Aladdin, USA), tetrahydrofuran (THF, $\geqslant 99 \%$, Tianjin Fuyu Fine Chemical Company), n-hexane (analytical grade, Tianjin Damao Chemical Reagent Company) and toluene (analytical grade, Laiyang Economic and Technological Development Zone Fine Chemical Factory) were also used in this experiment.

The UV-Vis spectra were treated with a Shimadzu UV-visible spectrophotometer UV-2550 equipped with Shimadzu/UV Probe software. A NicoletMAGNAIR550 (series II) spectrophotometer (USA) linked with Thermo Nicolet Corporation OMNIC32 software was used to export the data of Fourier transform infrared (FTIR) spectroscopy. The molecular weight $\left(M_{\mathrm{n}}\right)$ and molecular weight distribution $\left(M_{w} / M_{n}\right)$ of PGMA and PAN were recorded via a Waters 1515 gel permeation chromatograph (GPC) system consisted of a refractive-index detector (Waters 2414), using HR 1, HR 2 and HR $4(7.8 \times 300 \mathrm{~mm}, 5 \mu \mathrm{m}$ beads size $)$ columns with Mn ranging from $5 \times 10^{3}$ $6 \times 10^{5} \mathrm{~g} / \mathrm{mol}$. DMF was used as the eluent with a flow rate of $1.0 \mathrm{~mL} / \mathrm{min}$ at $35^{\circ} \mathrm{C}$. GPC samples were calibrated with poly(methyl methacrylate) standards obtained from Waters. A Germany Netzsch sta409pc luxx was applied to the mass loss determination of the polymers at a heating rate of 10 $\mathrm{K} / \mathrm{min}$ with $20-30 \mathrm{mg}$ sample under nitrogen. ${ }^{1} \mathrm{H}$ NMR spectrum of the polymers was recorded via an INOVA 400-MHz nuclear magnetic resonance instrument. The $\mathrm{CDCl}_{3}$ was used as a solvent, and the internal standard was tetramethylsilane (TMS).

\section{The synthesis of the RAFT agent 4-Cyanopentanoic Acid Dithiobenzoate (CPADB)}

$4.25 \mathrm{~g}$ dry di(thiobenzoyl) disulfide, $5.84 \mathrm{~g}$ dry 4,4'-azobis(4-cyanopentanoic acid) and $80 \mathrm{~mL}$ distilled ethyl acetate were combined in a $250 \mathrm{~mL}$ round-bottomed flask. Soon afterwards, a heating reaction of the solution was performed for $18 \mathrm{~h}$ under refluxing. The crude product was separated with the residual ethyl acetate in vacuo. With ethyl acetate and hexane (2:3) as eluent, a column chromatography (silicagel $60 \AA$, 70-230 mesh) was employed to purify the crude product. The combined red fractions were produced in column chromatography and dried all night with anhydrous sodium sulfate as desiccant. After removing solvent in vacuo, the red oily residue was placed at $-20{ }^{\circ} \mathrm{C}$ to crystallize. The target compound was obtained after recrystallizing from benzene. ${ }^{1} \mathrm{H}$ NMR (solvent $\left.\mathrm{CDCl}_{3}\right)$ (ppm): $7.91(2 \mathrm{H}, \mathrm{o}-\mathrm{Ar}), 7.60(1 \mathrm{H}, \mathrm{p}-\mathrm{Ar}), 7.42(2 \mathrm{H}, \mathrm{m}-\mathrm{Ar}), 2.40-2.80(4 \mathrm{H}$, $\left.\mathrm{CH}_{2} \mathrm{CH}_{2}\right), 1.98\left(3 \mathrm{H}, \mathrm{CH}_{3}\right)$.

\section{Typical RAFT polymerization of Polyacrylonitrile (PAN)}

In a $6 \mathrm{~mL}$-ampoule, CPADB (32.0 mg, $0.115 \mathrm{mmol}$ ) as RAFT agent, AIBN (9.40 mg, 0.0573 mmol; CTA/initiator molar ratio $=2)$ as initiator and acrylonitrile $(1.5 \mathrm{~mL}, 22.9 \mathrm{mmol})$ as monomer were dissolved with DMF $(3 \mathrm{~mL}$, solvent/monomer volume ratio $=2)$. Then the ampoule was placed into ice-water bath to be purged with dry nitrogen for $10 \mathrm{~min}$ for the thorough elimination of oxygen and water. Soon afterwards, the sealing of the ampoule was carried out via flame. A stirred water bath equipped with a thermostat was used as the place for the reaction to perform. The reaction was carried out at $60{ }^{\circ} \mathrm{C}$ for $8 \mathrm{~h}$. Then the ampoule with the product was cooled to room temperature, thereafter be broken open. The solution was totally precipitated in a large amount of methyl alcohol after the product was diluted with DMF. The polymer was separated by vacuum suction filtration and dried under vacuum until a constant weight at ambient temperature. Ultimately, the conversion of the polymer from monomer was determined by gravimetrical calculation.

Typical ATRP polymerization of Poly(glycidyl methacrylate) (PGMA)

Glycidyl methacrylate $(2 \mathrm{~mL}, 15.1 \mathrm{~mol})$ as monomer, EBIB $(22.3 \mathrm{uL}, 0.151 \mathrm{mmol})$ as initiator, $\mathrm{CuBr}_{2}(33.8 \mathrm{mg}, 0.151 \mathrm{mmol})$ as catalyst, PMDETA $(32.2 \mathrm{uL}, 0.151 \mathrm{mmol})$ and Vc $(26.6 \mathrm{mg}, 0.151$ 
mmol) as reductant were sequentially added into a 6 mL-ampoule with Toluene $(1 \mathrm{~mL}$, solvent/monomer volume ratio $=1$ ). As in the above experiment, the ampoule was also underwent the process of the thorough elimination of oxygen and water. Thereafter, the ampoule was burned to be sealed. And the ampoule was put in a stirred water bath equipped with a thermostat to carry out the experiment at $25{ }^{\circ} \mathrm{C}$ for required time. The ampoule was took out and cooled to room temperature when the reaction finished. Then the ampoule was broken open. The product was fully diluted with THF. Soon after, the solution was completely precipitated in a large amount of $\mathrm{n}$-hexane. The polymer was purified by vacuum filter and dried under vacuum to a constant weight at room temperature. Ultimately, the conversion of the polymer from monomer was determined by gravimetrical calculation.

\section{The synthesis of the poly(acrylonitrile-g-glycidyl methacrylate) brushes}

The modification of PAN with ammonium chloride as catalyst and in the presence of sodium azide was performed in a stirred oil bath equipped with a thermostat. A total of $53 \mathrm{mg}$ of PAN solid was dissolved with about $1 \mathrm{~mL}$ of DMF in a $6 \mathrm{~mL}$-ampoule. The mixture was stirred until it was well-distributed at ambient temperature. Subsequently, $65 \mathrm{mg}$ of $\mathrm{NaN}_{3}$ and $53.5 \mathrm{mg}$ of $\mathrm{NH}_{4} \mathrm{Cl}$ were added to the PAN solution with stirring. The ampoule was immediately put into an oil bath with stirring when the oil bath was heated to $120^{\circ} \mathrm{C}$. Then the temperature of the oil bath was held in $120^{\circ} \mathrm{C}$ for $12 \mathrm{~h}$. The final obtained reaction mixture was poured into a $4 \mathrm{~mL} \mathrm{H}_{2} \mathrm{O}$ and mixed thoroughly. Afterwards, $1 \mathrm{~mL}$ of the products were mixed with $1 \mathrm{~mL} \mathrm{DMF}$ and $1 \mathrm{~mL}$ triethylamine. Then $42.4 \mathrm{mg}$ of PGMA was thoroughly dissolved in the mixed liquor. The reaction mixture was continuously stirred for $3 \mathrm{~h}$ at $60^{\circ} \mathrm{C}$ to obtain the polymer brushes of poly(acrylonitrile-g-glycidyl methacrylate). Finally, the reaction mixture was loaded in dialysis bag to receive repeated dialysis. The end product was obtained after dried in low temperature by the vacuum freeze dryer.

\section{Results and Discussion}

\section{The polymerization of $P A N$ and $P G M A$}

The PAN was prepared via solution RAFT polymerization in the presence of AIBN and $\mathrm{CPADB}$. The polymerization carried out in DMF followed the under reaction conditions: $[A N] 0:[C P A D B] 0:[A I B N] 0=200: 1: 0.5, \quad A N: D M F=1: 2$. The fist-order kinetic plots for the RAFT polymerization of PAN were shown in Figure 1. As expected, the conversion of the monomer increased significantly with an increase of reaction time. And there is a well-liner relationship between $\ln ([\mathrm{M}] 0 /[\mathrm{M}])\left(\ln ([\mathrm{M}] / /[\mathrm{M}])=k_{\mathrm{p}}\right.$ app $\left.[\mathrm{R} \cdot] \mathrm{t}\right)$ and reaction time. These can intuitively indicate that the polymerization obeys the first-order kinetics. It obviously showed the controlled/"living" characteristic of the RAFT polymerization for PAN. However, Figure 1(a) also showed an inhibition period without polymerization activity for about $1.5 \mathrm{~h}$. This phenomenon can not only lower reactivity of the reinitiating group and monomer but also slow fragmentation rate of the pre-equilibrium RAFT radical to effect the polymerization. On the other hand, the influence for the relationship of conversion and molecular weight due to the inhibition period was very little. The plots of the number-average molecular weight $\left(M_{n}\right)$ and molecular weight distributions $\left(M_{w} / M_{n}\right)$, were shown in Figure 2, indicated that the $M_{n}$ of PAN increased linearly with the monomer conversion and kept close with the theoretical molecular weights. As it is shown in Figure 2(b), the $M_{w} / M_{n}$ values remained quite low. 
(a)

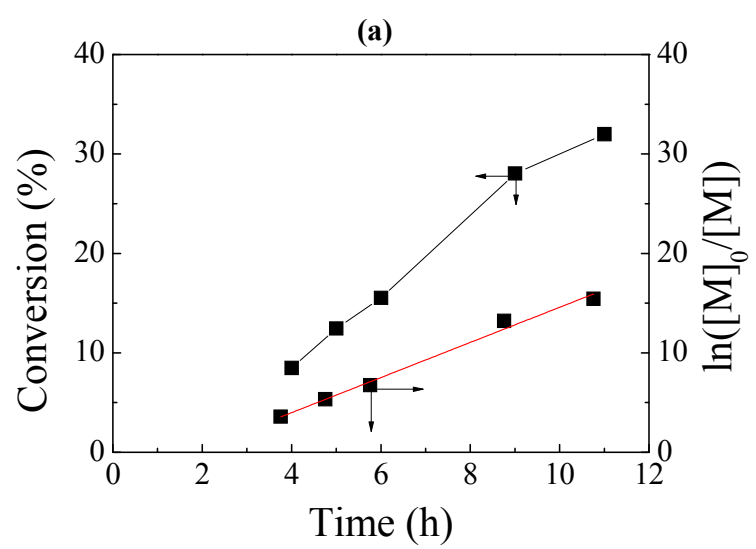

(b)

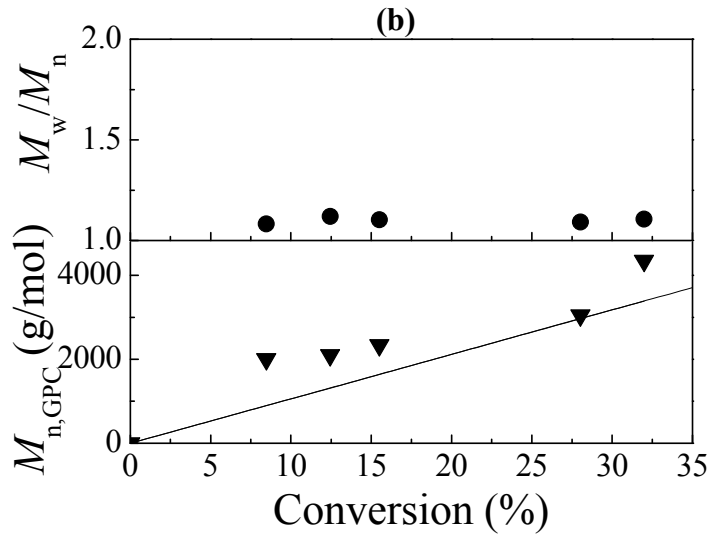

Figure 1. (a) First-order kinetics investigation of PAN with DMF as the solvent at $60{ }^{\circ} \mathrm{C}$. $[\mathrm{AN}]_{0}:[\mathrm{CPADB}]_{0}:[\mathrm{AIBN}]_{0}=200: 1: 0.5, V_{\mathrm{AN}}: V_{\mathrm{DMF}}=1: 2$. (b) Number-average molecular weight $\left(M_{n}\right)$ and molecular weight distribution $\left(M_{w} / M_{n}\right)$ vs monomer conversion for the RAFT polymerization of PAN.

The solution ATRP polymerization of PGMA was performed in toluene with EBIB as initiator, $\mathrm{CuBr}_{2} / \mathrm{PMDETA}_{\mathrm{P}}$ as the catalyst system and $\mathrm{V}_{\mathrm{c}}$ as reductant ([GMA]0:[EBiB]0:[CuBr2]0:[PMDETA $\left.]_{0}:\left[V_{c}\right] 0=100: 1: 1: 1: 1, \quad V_{\mathrm{GMA}}: V_{\text {Toulene }}=1: 1\right)$. The solution ATRP polymerization data of PGMA was summarized in Figure 2(a) and Figure 2(b). The fist-order kinetic plots visually indicated that the polymerization ran smoothly and strictly obeyed the first-order kinetics with $0.588 \mathrm{~h}^{-1}$ apparent rate constant of propagation $\left(k_{\mathrm{p}} \mathrm{app}\right)$. Namely, the number of radicals take part in the solution ATRP polymerization was persistent $\left(\ln \left([\mathrm{M}]_{0} /[\mathrm{M}]\right)=k_{\mathrm{p}} \mathrm{app}[\mathrm{R} \cdot] \mathrm{t}\right)$. Furthermore, the bi-radical termination reaction could ignore in this polymerization. As it is shown in Figure 2(a) and Figure 2(b), the obtained PGMA possessed well-controlled molecular structure and comparatively low molecular weight distributions $\left(M_{w} / M_{n}<1.6\right)$. It also can be seen in Figure 2(b), the molecular weight of PGMA measured via GPC was increased linearly versus the conversion of monomer and closed with the theoretical values.
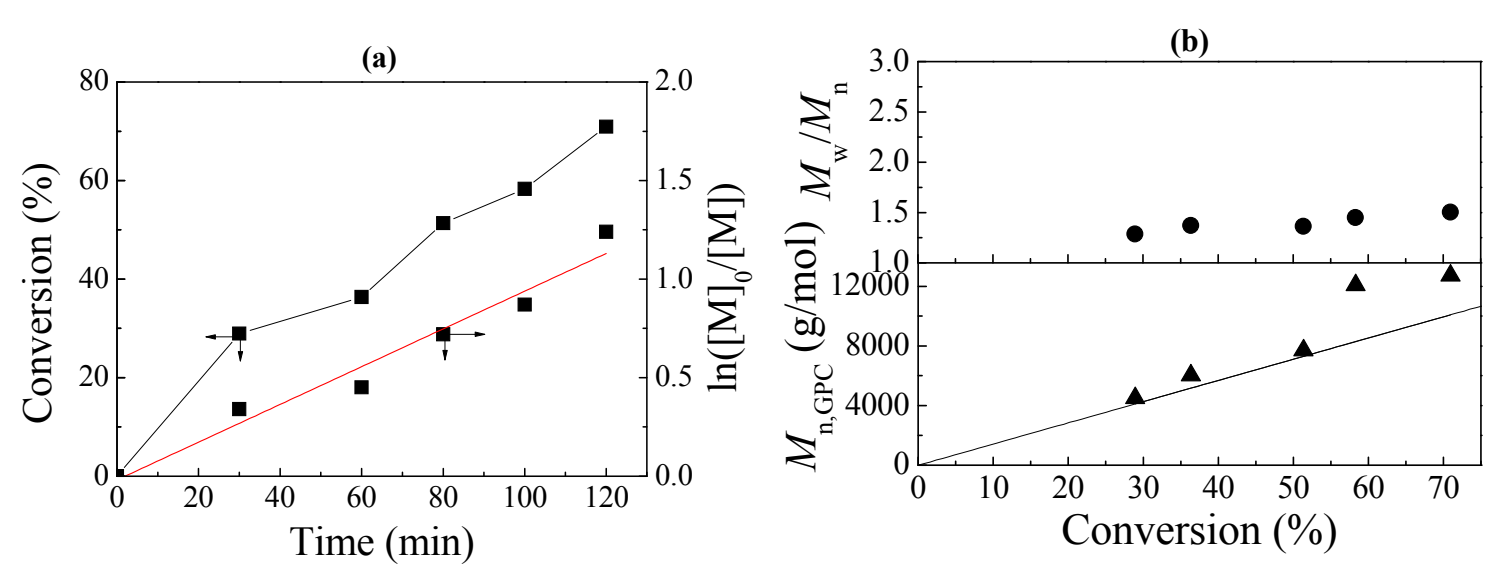

Figure 2. (a) First-order kinetics investigation of PGMA with toluene as the solvent at $25{ }^{\circ} \mathrm{C}$. $[\mathrm{GMA}]_{0}:[\mathrm{EBiB}]_{0}:\left[\mathrm{CuBr}_{2}\right]_{0}:[\mathrm{PMDETA}]_{0}:\left[\mathrm{VC}_{0}=100: 1: 1: 1: 1, V_{\mathrm{GMA}}: V_{\text {Toulene }}=1: 1\right.$. (b) Number-average molecular weight $\left(M_{n}\right)$ and molecular weight distribution $\left(M_{w} / M_{n}\right)$ vs monomer conversion for the ATRP polymerization of PGMA.

\section{Optimization of experimental conditions of the modification of PAN}

The "click chemistry" reaction of PAN leading to poly(5-vinyltetrazole) (PVT) was carried out for the synthesis of poly(acrylonitrile-g-glycidyl methacrylate) polymer brushes. The IR spectra for PAN and the obtained PVT were shown in Figure 3. PAN and PVT all exhibited a peak at 3700-3000 
$\mathrm{cm}^{-1}$ corresponding to the stretching vibration of $\mathrm{O}-\mathrm{H}$ by reason of the remaining water. A strong peak of PAN and PVT at $2925 \mathrm{~cm}^{-1}$ associated with asymmetric $\mathrm{CH}_{2}$ stretching vibration. A strong peak in PAN at $1666 \mathrm{~cm}^{-1}$ shifted to a weak wave in PVT at $1627 \mathrm{~cm}^{-1}$, which was attributed to the $\mathrm{C}=\mathrm{N}$ stretching vibration of PVT. In addition, a strong peak of PVT at $1452 \mathrm{~cm}^{-1}$ assigned to the characteristic $\mathrm{C}-\mathrm{H}$ bending vibration was weaker than the peak of PAN. The FTIR spectra of PAN exhibits characteristic PAN bands at $2243 \mathrm{~cm}^{-1}$ due to the $C \equiv N$ stretching vibration, disappears with click chemistry reaction, indicates that $\mathrm{C} \equiv \mathrm{N}$ groups have reduced into tetrazole groups. Therefore, the FT-IR spectra of PAN and PVT proved the formation of PVT.

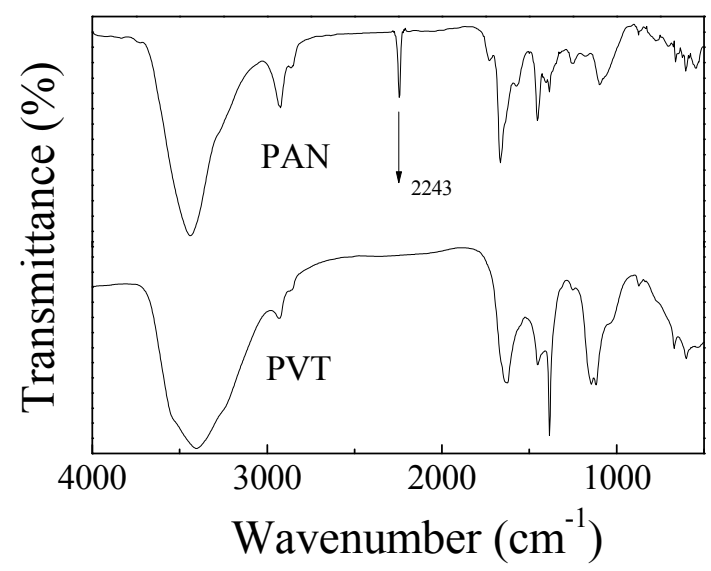

Figure 3. The FT-IR spectra of PAN and PVT.

Figure 4 displayed the UV-Vis spectra of the obtained PVT modified from PAN $\left(M_{\mathrm{w}}=9000\right.$ $\mathrm{g} / \mathrm{mol}$ ) for the reaction time of 7, 8, 9 and $12 \mathrm{~h}$. As shown in Figure 6, a strong absorption at around $282 \mathrm{~nm}$ corresponding to $\mathrm{n}-\pi^{*}$ absorption was exhibited in the four PVTs due to the presence of tetrazole rings. Specifically, in addition to the much stronger absorption at $282 \mathrm{~nm}$, the PVT modified for $12 \mathrm{~h}$ also exhibited an extra absorption at $338 \mathrm{~nm}$. These results obtained from UV-vis spectra were assigned to the presence of aromatic tetrazole group in PVT modified from PAN with the participation of sodium azide and ammonium chloride. In addition, the UV-vis spectra also suggested that the absorption increased with the increasing of reactive time. It may be attributed to the change of the reaction degree. Thus, $12 \mathrm{~h}$ was chosen as the optimal reactive time.

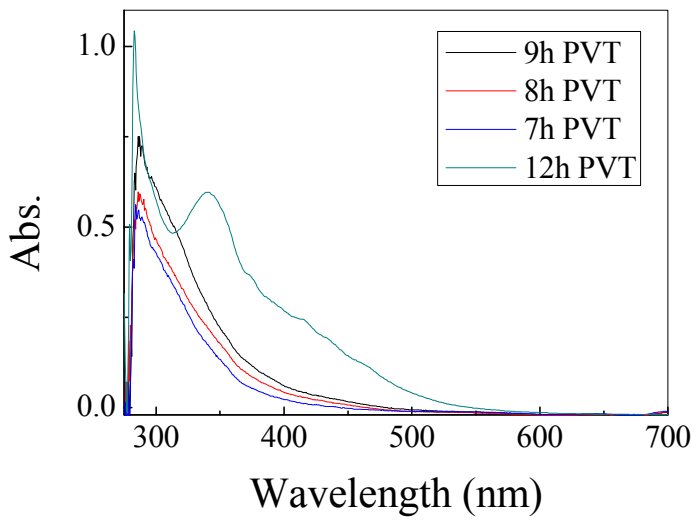

Figure 4. UV-vis spectra of PVT with different reaction time.

The modification of PAN was related to the ratio of reactant. In order to optimize the ratio of PAN, sodium azide and ammonium chloride, a series of experiment with the ratio of 1:0.1:0.1, 1:0.3:0.3, 1:0.4:0.4, 1:0.5:0.5, 1:1:1 was carried out. Early on in the formation of tetrazole ring, the directly use of hydrazoic acid was the best choice consider of the convenience. However, hydrazoic acid was replaced soon with other acids in union with a source of azide due to its highly toxicity and explosibility. As is shown in Scheme 1, the "click chemistry" reaction in this experiment was 
the 1,3-dipolar cycloaddition of PAN with sodium azide as the source of azide and ammonium chloride as catalyst. Figure 5 displayed the UV-vis spectra of the obtained PVT modified from PAN $\left(M_{\mathrm{w}}=9000 \mathrm{~g} / \mathrm{mol}\right)$ for the ratio of 1:0.1:0.1, 1:0.3:0.3, 1:0.4:0.4, 1:0.5:0.5, 1:1:1. When the ratio of $\mathrm{n}: 1: 1$, the absorption coefficient of PVT was the maximum, the UV-vis spectra suggesting that the ratio of 1:1:1 was the optimal ratio. In addition, the FT-IR spectra proved that the PAN was totally modified into PVT in the ratio of 1:1:1 for $12 \mathrm{~h}$. As a result, the modified reaction was carried out in the condition that the ratio of PAN, sodium azide and ammonium chloride was 1:1:1 and the reactive time was $12 \mathrm{~h}$.

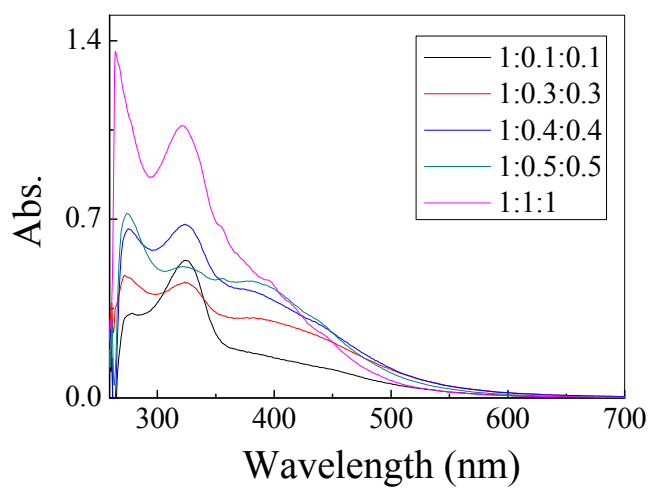

Figure 5. UV-vis spectra of PVT with the different ratio of PAN, sodium azide and ammonium chloride (1:0.1:0.1, 1:0.3:0.3, 1:0.4:0.4, 1:0.5:0.5, 1:1:1).

Characterization of poly(acrylonitrile-g-glycidyl methacrylate) polymer brushes

In addition, Figure 6 represented the 1H NMR spectrum of PGMA, PAN and the PAN-g-PGMA prepared via the combination of controlled/“living” radical polymerization and click chemistry. The chemical shift at around $1.27 \mathrm{ppm}$ (a) was assigned to the methyl protons of PGMA in the polymer brushes. The resonances at $2.00 \mathrm{ppm}(\mathrm{b})$ corresponded to the methylene protons of PGMA in the polymer brushes. At the same times, the signal of the side group in the PGMA was assigned to the peaks at around $3.66 \mathrm{ppm}$ at position $\mathrm{c}$ in the spectrum. The characteristic resonances originating from the oxirane ring of PGMA in the polymer brushes were assigned to the peaks at $3.22 \mathrm{ppm}(\mathrm{d})$ and position e of $2.78-2.59 \mathrm{ppm}$ and $2.96 \mathrm{ppm}$. Meanwhile, the two protons of PAN in the polymer brushes also gave two different resonances in the spectrum of Figure 6 . The signal at $2.00 \mathrm{ppm}$ (f) corresponding to methylene protons of PAN in the polymer brushes. And the signal at $3.15 \mathrm{ppm}(\mathrm{g})$ derives from the methylidyne protons of PAN in the polymer brushes. In summary, these chemical shifts clearly indicated the successful prepared of the polymer brushes.

(a)

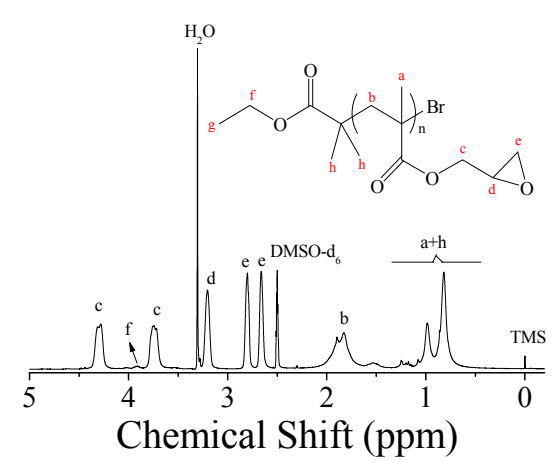

(b)

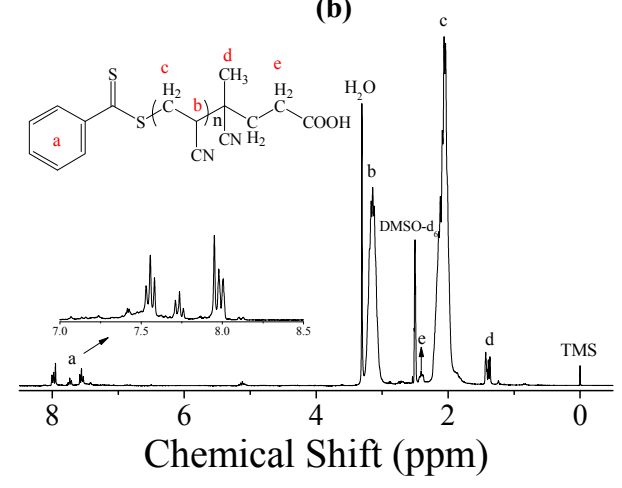




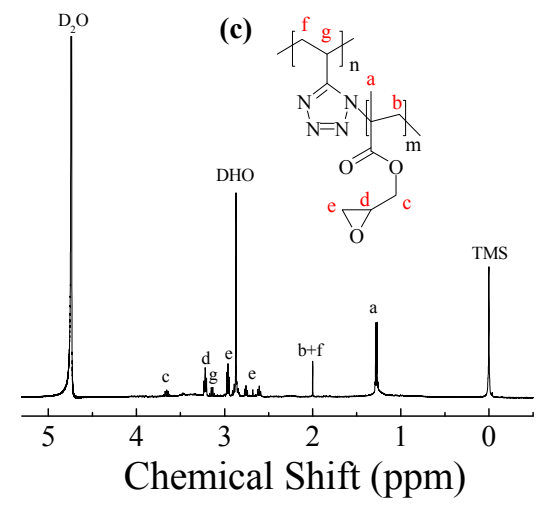

Figure 6. The ${ }^{1} \mathrm{H}$ NMR spectrum (INOVA $400 \mathrm{MHz}$ ) of PGMA (a), PAN (b) and the polymer brushes (c) recorded at $25{ }^{\circ} \mathrm{C}$ with $\mathrm{CDCl}_{3}$ and $\mathrm{D}_{2} \mathrm{O}$ as solvent.

For the formation of PGMA with unique terminal bromine, a series of ATRP polymerization was performed. The FTIR spectra of the PGMA obtained from ATRP polymerization was shown in Figure 7. The peaks about $3000-2840 \mathrm{~cm}^{-1}$ were assigned to stretching vibration of $\mathrm{CH}_{3}$ and $\mathrm{CH}_{2}$ in PGMA. A strong absorption is observed at $1731 \mathrm{~cm}^{-1}$ due to the $\mathrm{C}=\mathrm{O}$ stretching vibration in PGMA. The PGMA also exhibited the absorption at $1259 \mathrm{~cm}^{-1}$ and $1150 \mathrm{~cm}^{-1}$ assigned to stretching vibration of $\mathrm{O}=\mathrm{C}-\mathrm{O}$ and COO-C respectively due to the presence of ester group. The peak at $907 \mathrm{~cm}^{-1}$ was attributed to the characteristic of epoxy group in PGMA. On the other hand, Figure 9 also showed the FT-IR spectra of PAN and PAN-g-PGMA. PAN-g-PGMA exhibits bands at $1632 \mathrm{~cm}^{-1}$ and 1727 $\mathrm{cm}^{-1}$, which were attributed to the characteristic $\mathrm{C}=\mathrm{N}$ stretching vibration of the modified PAN and the characteristic $\mathrm{C}=\mathrm{O}$ stretching vibration of PGMA, respectively, demonstrated the successful preparation of polymer brushes.

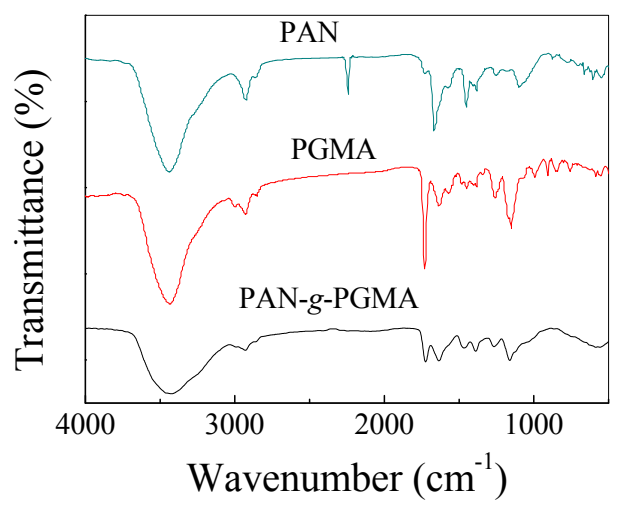

Figure 7. The FT-IR spectra of PAN, PGMA and PAN-g-PGMA.

For further investigation the synthesis of polymer brushes, a characterization by TGA was executed. The TGA curves of PAN, PGMA and Brush were compared in Figure 8. It is known from Scheme 1, the polymer brushes was composed with PAN as the linear polymer backbone and PGMA as densely grafted polymer side chains. In the TGA thermograms, the Brush underwent an additional $41 \%$ weight loss compared with PAN due to the presence of a number of PGMA. Moreover, the Brush started a major decomposition at a lower temperature than PAN and has a broader range of the process. These phenomenons provided strong evidence that the PAN-g-PGMA polymer brushes was successfully prepared. 


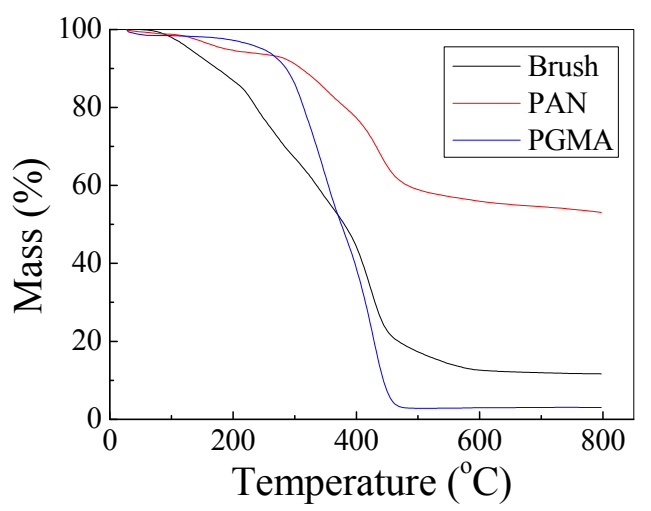

Figure 8. The TG spectrum of PGMA, PAN and PAN-g-PGMA.

\section{Conclusions}

PAN regarded as linear polymer backbone was prepared via the RAFT polymerization and PGMA regarded as densely grafted polymer side chains was prepared via the ATRP polymerization. The union of controlled/"living" radical polymerization and click chemistry as a new strategy enabled to successfully prepare a novel functional polymer brushes of poly(acrylonitrile-g-glycidyl methacrylate).

Acknowledgments: The financial was supported by the National Natural Science Foundation of China (Nos. 51573075, 21404051 and 21404052), the Natural Science Foundation of Shandong Province (Nos. ZR2014BQ016 and BS2014CL040), the Project of Shandong Province Higher Educational Science (Nos. J16LC20) and Technology Program and the Program for Scientific Research Innovation Team in Colleges and universities of Shandong Province.

\section{References}

1. B. Zhao, W. J. Brittain, Prog. Polym. Sci., 2000, 25, 677-710.

2. S. Edmondson, V. L. Osborne, W. T. Huck, Chem. Soc. Rev., 2004, 33, 14-22.

3. S. T. Milner, T. A. Witten, M. E. Cates, Macromolecules, 1988, 21, 2610-2619.

4. M. Joubert, C. Delaite, E. B. Lami, P. Dumas, New. J. Chem., 2005, 29, 1601-1609.

5. R. Djalali, N. Hugenberg, K. Fischer, M. Schmidt, Macromol. Rapid. Comm., 1999, 20, 444-449.

6. T. Stephan, M. A. Sandra, M. Schmidt, Macromolecules, 2002, 35, 9857-9860.

7. J. A. Johnson, Y. Y. Lu, A. O. Burts, Y. H. Lim, M. G. Finn, J. T. Koberstein, N. J. Turro, D. A. Tirrell, R. H. Grubbs, J. Am. Chem. Soc., 2011, 133, 559-566.

8. K. J. Yao, Y. Chen, J. Zhang, C. Bunyard, C. B. Tang, Macromol. Rapid. Comm., 2013, 34, 645-651.

9. B. S. Sumerlin, D. Neugebauer, K. Matyjaszewski, Macromolecules, 2005, 38, 702-708.

10. J. X. Ding, C. S. Xiao, Z. H. Tang, X. L. Zhuang, X. S. Chen, Macromol. Biosci., 2011, 11, 192-198.

11. J. P. Zhao, G. Z. Zhang, S. Pispas, J. Polym. Sci. Pol. Chem., 2010, 48, 2320-2328.

12. H. F. Gao, K. Matyjaszewski, J. Am. Chem. Soc., 2007, 129, 6633-6639.

13. 26. Y. C. Yan, Y. Shi, W. Zhu, Y. M. Chen, Polymer, 2013, 54, 5634-5642.

14. L. Zou, W. Zhu, Y. M. Chen, Polymer, 2013, 54, 481-484.

15. K. Matyjaszewski, J. Spanswick, Mater. Today., 2005, 8, 26-33.

16. M. F. Cunningham, Prog. Poly. Sci., 2008, 33, 365-398.

17. D. J. Siegwart, J. K. Oh, K. Matyjaszewski, Prog. Poly. Sci., 2012, 37, 18-37.

18. J. Ran, L. Wu, Z. H. Zhang, T. W. Xu, Prog. Poly. Sci., 2014, 39, 124-144.

19. K. Matyjaszewski K, Macromolecules, 2012, 45, 4015-4039.

20. K. L. Robinson, M. A. Khan, M. V. de Paz Báñez, X. S. Wang, S. P. Armes, Macromolecules, 2001, 34, 3155-3158.

21. S. H. Qin, D. Q. Qin, K. Y. Qiu, New. J. Chem., 2001, 25, 893-895.

22. A. Pourjavadi, M. Adeli, M. Yazdi, New. J. Chem., 2012, 37, 295-298.

23. L. Shen, C. Ma, S. Z. Pu, C. J. Cheng, J. K. Xu, L. Lia, C. Q. Fu, New. J. Chem., 2009, 33, 825-830. 
24. J. Chiefari, Y. K. (Bill) Chong, F. Ercole, J. Krstina, J. Jeffery, T. P. T. Le, R. T. A. Mayadunne, G. F. Meijs, C. L. Moad, G. Moad, E. Rizzardo, S. H. Thang, Macromolecules, 1998, 31, 5559-5562.

25. J. Brassard, M. Gottschalk, S. Quessy, Macromolecules, 2003, 36, 1505-1513.

26. C. Boyer, V. Bulmus, T. P. Davis, V. Ladmiral, J. Q. Liu, S. Perrier, Chem. Rev., 2009, 109, 5402-5436.

27. G. Moad, E. Rizzardo, S. H. Thang, Cheminform, 2013, 44.

28. Q. Zhang, Z. B. Zhang, W. X. Wang, J. Zhu, Z. P. Cheng, N. C. Zhou, W. Zhang, X. L. Zhu, J. Polym. Sci. Poly. Chem., 2012, 50, 1424-1433.

29. H. F. Gao, K. Matyjaszewski, Macromolecules, 2006, 39, 4960-4965.

30. J. F. Lutz, H. G. Börner, K. Weichenhan, Macromolecules, 2006, 39, 6376-6383.

31. R. Ranjan, W. J. Brittain, Macromol. Rapid. Comm., 2007, 28, 2084-2089.

32. H. C. Kolb, M. G. Finn, K. B. Sharpless, Angew. Chem., 2001, 113, 2056-2075.

33. H. Nandivada, X. Jiang, J. Lahann, Adv. Mater., 2007, 19, 2197-2208.

34. H. C. Kolb, K. B. Sharpless, Drug. Discov. Today., 2003, 8, 1128-1137.

35. J. E. Moses, A. D. Moorhouse, Cheminform, 2007, 38, 1249-1262.

36. P. Wu, M. Malkoch, J. N. Hunt, R. Vestberg, E. Kaltgrad, M. G. Finn, V. V. Fokin, K. B. Sharpless, C. J. Hawker, Chem. Commu., 2012, 37, 5775-5777.

37. F. Alonso F, Y. Moglie, G. Radivoy, Accounts. Chem. Res., 2015, 48, 2516-2528.

38. J. D. Megiatto, D. I. Schuster, New. J. Chem., 2010, 34, 276-286.

39. J. Zhan, D. Tian, H. Li, New. J. Chem., 2008, 33, 725-728.

40. G. Molteni, C. L. Bianchi, G. Marinoni, New. J. Chem., 2006, 30, 1137-1139.

41. V. V. Rostovtsev, L. G. Green, V. V. Fokin, K. B. Sharpless, Angew. Chem. Int. Edit., 2002, 41, 45.

42. H. Isobe, K. Cho, N. Solin, D. B. Werz, P. H. Seeberger, E. Nakamura, Org. Lett., 2007, 9, 4611-4614.

43. A. Scarpaci A, C. Cabanetos, E. Blart, V. Montembault, L. Fontaine, V. Rodriguez, F. Odobel, J. Poly. Sci. Poly. Chem., 2009, 47, 5652-5660.

44. M. Bourourou, M. Holzinger, F. Bossard, F. Hugenell, A. Maaref, S. Cosnier, Carbon, 2015, 87, $233-238$.

45. Y. Mitsukami, M. S. Donovan, A. B. Lowe, C. L. McCormick, Macromolecules, 2001, 34, 2248-2256. 